\title{
The Ultraviolet Absorption Coefficient of Melanosomes Decreases with Increasing Pheomelanin Content
}

\author{
Dana N. Peles and John D. Simon* \\ Department of Chemistry, Duke University, Durham, North Carolina 27708
}

Received: March 23, 2010; Revised Manuscript Received: May 28, 2010

\begin{abstract}
Uveal melanosomes from the iridal stroma contain both eumelanin and pheomelanin, the ratio of which varies with iris color. Herein, we report the absorption coefficient at $\lambda=244 \mathrm{~nm}$ for individual human iridal stroma melanosomes from dark brown and blue-green irides. The melanosomes are nearly identical in size, but differ in the relative concentration composition, ranging from a eumelanin/pheomelanin ratio of 14.8:1 (dark brown) to 1.3:1 (blue-green or hazel). The absorption coefficient of the melanosome decreases as its pheomelanin content increases. The origin of this decrease is attributed to a corresponding decrease in the number of UV-absorbing chromophores, reflecting the different molecular volumes of the monomeric building blocks of the two pigments. In agreement with reported data on synthetic pigments, the absorption coefficient of pheomelanin is found to be slightly larger than that for eumelanin at $\lambda=244 \mathrm{~nm}$ (by a factor of 1.2). On the basis of the reported optical properties of synthetic models, this result suggests that the absorption of pheomelanin is less than eumelanin at wavelengths of biological relevance $(\sim 315-400 \mathrm{~nm})$.
\end{abstract}

\section{Introduction}

Melanin is a natural pigment that is generally produced within organelles (melanosomes) that are located in pigment-producing cells called melanocytes. ${ }^{1-4}$ Typically, melanins are categorized into two groups: eumelanin (black-brown, derived from 5,6dihydroxy-indole, DHI; and 5,6-dihydroxy-indole-2-carboxylic acid, DHICA) and pheomelanin (red-yellow, derived from isomers of cysteinyl dopa). ${ }^{5-7}$ Mixtures of these pigments are widely produced within melanosomes through in vivo melanogenesis, although some noted exceptions are the pure eumelanic melanosomes produced in black hair and the retinal pigment epithelium of the eye. ${ }^{4,8,9}$ The biological functions of melanins are often attributed to their unique chemical properties; however, their molecular structure and organization within the melanosome remains largely unknown.

Epidemiological data for uveal melanoma, the most common intraocular malignant tumor in human adults, suggests a link between incidence and the relative concentrations of pheomelanin. ${ }^{10,11}$ This observation is consistent with studies that indicate that pheomelanin is more pro-oxidant than eumelanin. ${ }^{12-17}$ Determining the quantum efficiency for oxygen activation by these different melanins, however, requires knowing their relative absorption coefficient. Unfortunately, one has not been able to determine the absorption coefficient for eumelanin and pheomelanin contained in intact melanosomes, and so this information is generally obtained from the study of synthetic model systems dissolved or suspended in buffer solutions that mimic physiological conditions. Furthermore, since the molecular structure of melanins remains elusive, the absorption coefficients, $\varepsilon$, are usually expressed in units of $\mathrm{cm}^{-1}(\mathrm{mg} / \mathrm{mL})^{-1}$.

We recently demonstrated that the photoemission electron microscope (PEEM) could be used to directly quantify the absorption coefficient of single, intact melansomes. ${ }^{18}$ Herein, we used this approach to report the first measurement of the absorption coefficients of intact iridal stroma melanosomes

* To whom correspondence should be addressed. E-mail: john.simon@ duke.edu. isolated from dark brown and blue-green human irides. Iridal stroma melanosomes are an excellent system for quantifying the natural absorption coefficients of the two classes of pigments (eumelanin and pheomelanin) because different colored irides produce organelles of varying eumelanin/pheomelanin ratios with similar size and morphology. Chemical degradation analyses reveal eumelanin/pheomelanin ratios of 14.8 and 1.3 for melanosomes present in dark brown and blue-green human irides, respectively, ${ }^{10}$ and therefore, this single natural system enables a systematic approach for determining how the absorption coefficient changes with varying pigment composition.

\section{Experimental Methods}

Sample Isolation and Preparation. Details regarding the isolation of the human iridal stroma melanosomes used in this study have been previously published; ${ }^{19}$ these samples were the same that were used to determine the iridal stroma melanosome size distributions and surface photoionization properties. ${ }^{19}$ Briefly, irises were extracted from donor eyes of two patients of different colors and ages, dark brown, age 14 and blue-green, age 66. The irises were isolated from the iridal pigment epithelium through a previously described method. ${ }^{20}$ Following this step, the procedure reported for the enzymatic extraction of melanosome isolation from bovine irides ${ }^{21}$ was utilized to isolate the iridal stroma melanosomes from the iris tissue. In this method, small pieces of wet iris tissue were enzymatically digested at $37{ }^{\circ} \mathrm{C}$ in PBS under argon in three steps: collagenase (68 U/mL, with $2 \mathrm{mM} \mathrm{CaCl}_{2}$ ) for $\sim 16 \mathrm{~h}$, pancreatin $(2.5 \mathrm{mg}$ / $\mathrm{mL})$ for $2 \mathrm{~h}$, and trypsin $\left(646 \mathrm{U} / \mathrm{mL}\right.$, with $\left.2 \mathrm{mM} \mathrm{CaCl}_{2}\right)$ for $2 \mathrm{~h}$. Following each digestion, the remaining material was washed with water several times. After these treatments, the materials were suspended in $1 \%$ (wt/vol) Triton X-100 solution and stirred for $1.5 \mathrm{~h}$ at room temperature under argon. Subsequently, the particles were washed with methanol/water $1: 1(\mathrm{v} / \mathrm{v})$ once and then with water four times. Afterward, the resulting pellets were subjected to another iteration of the enzymatic digestion with collagenase $(68 \mathrm{U} / \mathrm{mL}$, with $2 \mathrm{mM}$ $\mathrm{CaCl}_{2}$ ) for $16 \mathrm{~h}$ at $37^{\circ} \mathrm{C}$ under argon. The resulting black to 

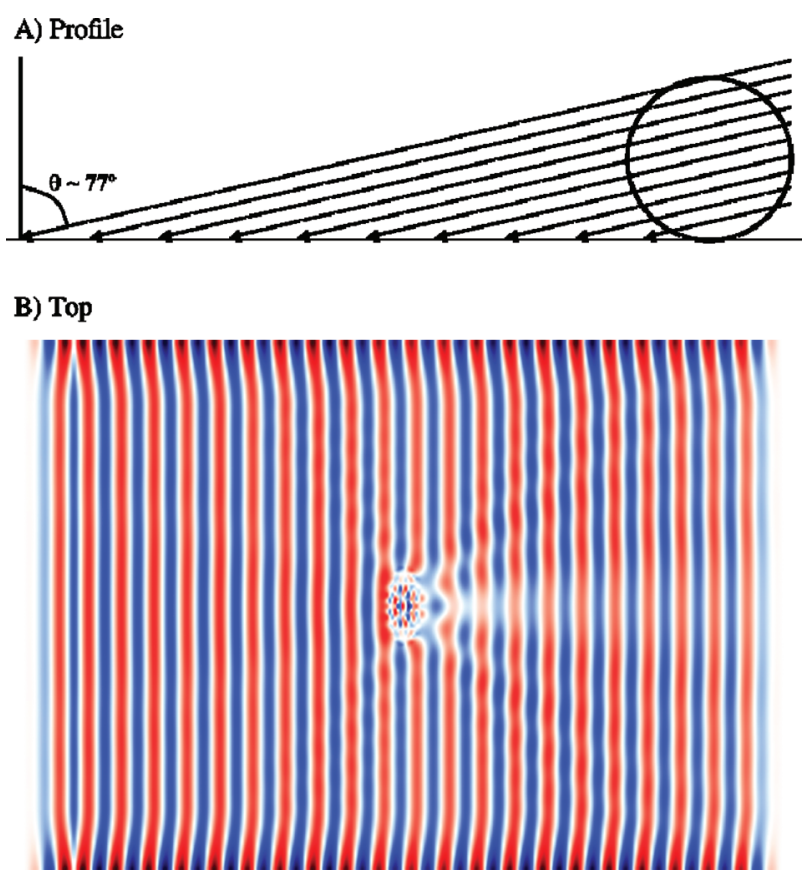

Figure 1. (A) An experimental schematic of the incident light pathlengths through an object in the PEEM system. (B) A top view $(x-y$ dimension $)$ simulation of the interaction of electromagnetic radiation $(\lambda=244 \mathrm{~nm})$ with an ellipsoid (model for the melanosome) characterized with a refractive index of 3.4. The dimensions of the ellipsoid are comparable to the melanosomes studied herein $(260 \mathrm{~nm}$ $\times 560 \mathrm{~nm}$ ). Simulations of the electromagnetic field were performed with the finite-difference time-domain method ${ }^{56}$ using a freely available software package. ${ }^{57}$ The colors reflect the peaks and valleys of the electromagnetic radiation.

brown melanosomes were then washed with water five times and stored at $-70{ }^{\circ} \mathrm{C}$ until use.

Photoemission Electron Microscopy. Details of the Elmitec ultrahigh vacuum PEEM used herein have been previously described in detail. ${ }^{22}$ Substrates were prepared from wafers of $\langle 100\rangle$-oriented silicon (P-type, B-doped), purchased from VA Semiconductor, Inc. (Fredericksburg, VA). These wafers were cleaved to square regions of approximately $1 \mathrm{~cm}^{2}$ and then cleaned using a simple solvent procedure. Specifically, wafers were added, in subsequent steps, to a trichloroethylene bath, an acetone bath, and a methanol bath for 5 min each. The wafers were then placed on lens paper in a sterile Petri dish and dried with a steady flow of nitrogen gas. Afterward, the cleaned wafers

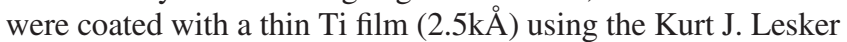
PVD 75 E-beam and Thermal Metal Evaporator (Pittsburgh, PA). Isolated melanosomes were then suspended in a Nanopure water solution for deposition on the experimental substrate. These suspensions were deposited onto the Ti-coated wafers in $0.5 \mu \mathrm{L}$ aliquots immediately prior to experimentation. The samples were air-dried $(<1 \mathrm{~h})$ with minimal exposure to ultraviolet light and then subsequently transferred under ultrahigh vacuum into the PEEM chamber for data collection. Melanosomes were imaged at fields of view of $50 \mu \mathrm{m}$ to ensure a large spatially resolved sampling. All images were captured with a cooled DVC-1500 M digital camera from DVC Company, Inc. (Austin, TX). The resolution of the digital camera was $1392 \times 1040$ pixels $\times 12$ bits, and the acquisition time was $20 \mathrm{~s}$.

Laser excitation of the sample at $\lambda=244 \mathrm{~nm}(5.07 \mathrm{eV})$ was provided by an intracavity doubled $\mathrm{Ar}^{+}$ion laser. A zero-order half-wave plate was used to orient the polarization of the light parallel to the plane of incidence. The laser was focused on the sample at an angle of incidence of approximately $77^{\circ}$ (with respect to the surface normal), as shown in Figure $1 \mathrm{~A}$. The Ti substrate and the melanosomes have work functions of 4.33 and $\sim 4.9 \mathrm{eV}$, respectively, and thus, the energy of the incident photon beam exceeded the threshold ionization potential of both. ${ }^{12,23}$ The efficiency of ionization (electrons per area) differs between the substrate and the melanosome, so the organelles can be resolved in the image.

As light travels though the melanosome, absorption attenuates its intensity, causing a reduction in the photoemission of the substrate immediately behind the melanosome, and a shadow is visualized as a result. However, as simulated in Figure 1B, illumination of an ellipsoidal object with $\lambda=244 \mathrm{~nm}$ radiation produces significant scattering of the electric field. Consequently, the absorptive properties of the melanosomes, which are manifested by changes in the image intensity of the substrate, are determined from the region of the substrate immediately behind the melanosome that is void of significant scattering contributions.

Determining the Absorption Coefficient from the PEEM Image. The absorption coefficient, $\varepsilon c$, is defined by the Beer-Lambert Law:

$$
\varepsilon \mathrm{c}=A l^{-1}=l^{-1} \log \left(I_{0} / I\right)
$$

The quantity $\varepsilon c$ has units of inverse length. Its calculation requires knowledge of the path length, $l$, and the incident and transmitted intensities of light, $I_{0}$ and $I$, respectively. The values of $I$ and $I_{0}$ are derived from the PEEM image; an average of the photoemission current of the substrate, where there is no obstruction by absorbing melanosomes, gives $I_{0}$, and an average of the photoemission current of the shadow region adjacent to the melanosomes gives $I$. In principle, the spatial dependence of the photoemission current of the shadow maps directly onto the different pathlengths through the melanosome. In practice, however, it is only possible to precisely know the dimensions of the melanosome to within a few tens of nanometers, the resolution of the PEEM. This uncertainty covers the majority of the range of the size distribution of the sample as determined by $\mathrm{SEM},{ }^{19}$ so the following approach was developed to determine $\varepsilon \mathrm{c}$ from average values for the melanosome dimensions and shadow intensity.

Scanning electron microscopy and atomic force microscopy reveal that human iridal stroma melanosomes are ellipsoidal. ${ }^{19}$ As a result, points in the shadow correspond to different chord lengths through the ellipsoid. To compute the absorption coefficient, we determined an average path length and used that value in eq 1 along with the average photoemission intensity obtained from the shadow and the unobstructed substrate regions. The average path length calculation is derived as follows. Consider an ellipsoid described by

$$
\left[\left(x^{2} / a^{2}\right)+\left(y^{2} / b^{2}\right)+\left(z^{2} / c^{2}\right)\right]=1
$$

where $a$ and $b$ are the equatorial radii along the $x$ - and $y$-axes, respectively (in the plane of the substrate), and $c$ is the polar radius along the $z$-axis (normal to the substrate), Figure 2. Solving this equation for $y$ produces two functions that correspond to the two parabolas of a 2-D horizontal ellipse at a height, $z$, shown in Figure 2. The path length, $s$, between points 


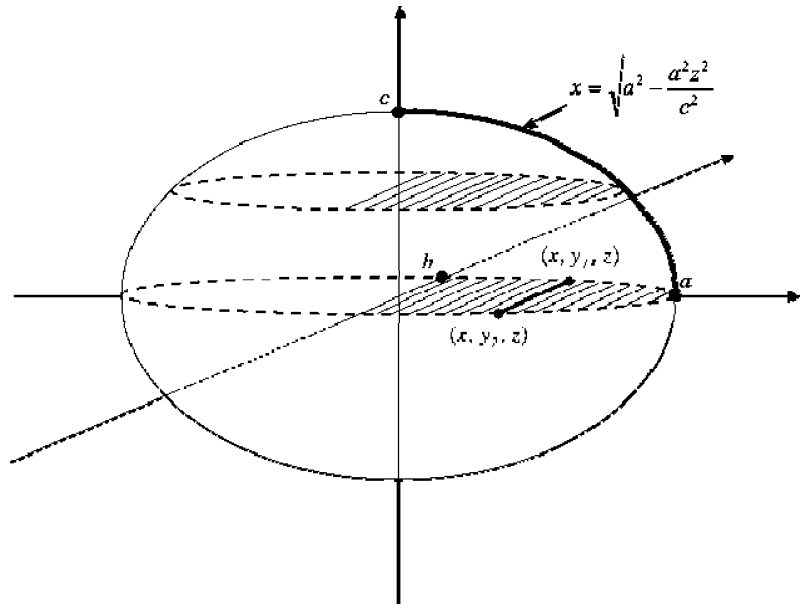

Figure 2. Geometrical model of an ellipsoid with dimensions $a, b$, and $c$. Parabolas are diagramed within the ellipsoid as the two halves of the dashed 2-D ellipses. An example of a single path length, $s$, passing through the ellipse is highlighted in black between the points $\left(x, y_{1}, z\right)$ and $\left(x, y_{2}, z\right)$. The equation for the highlighted curvature of a melanosome along the $x$-axis is shown.

on each parabola as a function of $x$, and $z$ can thus be calculated from

$$
s(x, z)=2\left\{b^{2}-\left[\left(b^{2} x^{2}\right) / a^{2}\right]-\left[\left(b^{2} z^{2}\right) / c^{2}\right]\right\}^{1 / 2}
$$

To find the average distance of all of the pathlengths of the collection of 2-D ellipses that make up the ellipsoid, integration with appropriate bounds is required. Consider, the 2-D ellipse at height $z=0$. Here, the range can be limited to $x \in[0, a]$ (by symmetry, the range can be limited to $a$ instead of $2 a$ ). However, as the height, $z$, changes for each 2-D ellipse, the range must be limited to the radius of that particular slice along the $x$-axis. Thus, with the range $z \in[0, c]$, it must be that $x \in\left[0,\left[a^{2}-\right.\right.$ $\left.\left.\left(a^{2} z^{2}\right) / c^{2}\right]^{1 / 2}\right]$ to account for the curvature of the ellipsoid. Using these bounds, the average path length of an ellipsoid is given by

$$
\bar{s}=\frac{\int_{0}^{c} \int_{0}^{\left[a^{2}-\left(a^{2} z^{2}\right) / c^{2}\right]^{1 / 2}} s(x, z) \mathrm{d} x \mathrm{~d} z}{\int_{0}^{c} \int_{0}^{\left[a^{2}-\left(a^{2} z^{2}\right) / c^{2}\right]^{1 / 2}} \mathrm{~d} x \mathrm{~d} z}
$$

It must be noted, however, that portions of the analyzed shadow regions generally map onto pathlengths that extend beyond half of the volume of the melanosome, but not the entire melanosome, due to the geometry of the experiment. The polar distance, $\chi$, that extends below $z=0$ and accounts for the additional pathlengths associated with the shadow region can be obtained directly from the PEEM image. Once $\chi$ is determined, the averaged experimental pathlength is calculated by integrating eq 4 with the integration bounds $z \in[\chi, \mathrm{c}]$ and $x \in\left[-a^{2}-\right.$ $\left.\left.\left(a^{2} z^{2}\right) / c^{2}\right]^{1 / 2},\left[a^{2}-\left(a^{2} z^{2}\right) / c^{2}\right]^{1 / 2}\right]$.

The above analysis is accurate for ellipsoids whose long axis is oriented perpendicular to the direction of incident light. However, in a typical sample, melanosomes are oriented at random angles with respect to the direction of incident light. Size analyses of the iridal stroma melanosomes reveal a long axis of $\sim 550 \mathrm{~nm}$ and an aspect ratio of $\sim 2 .{ }^{19}$ Unfortunately, with small dimensions and aspect ratios, determination of the angle between the long axis of the ellipsoid and the incident light is difficult to achieve with reasonable accuracy. In this

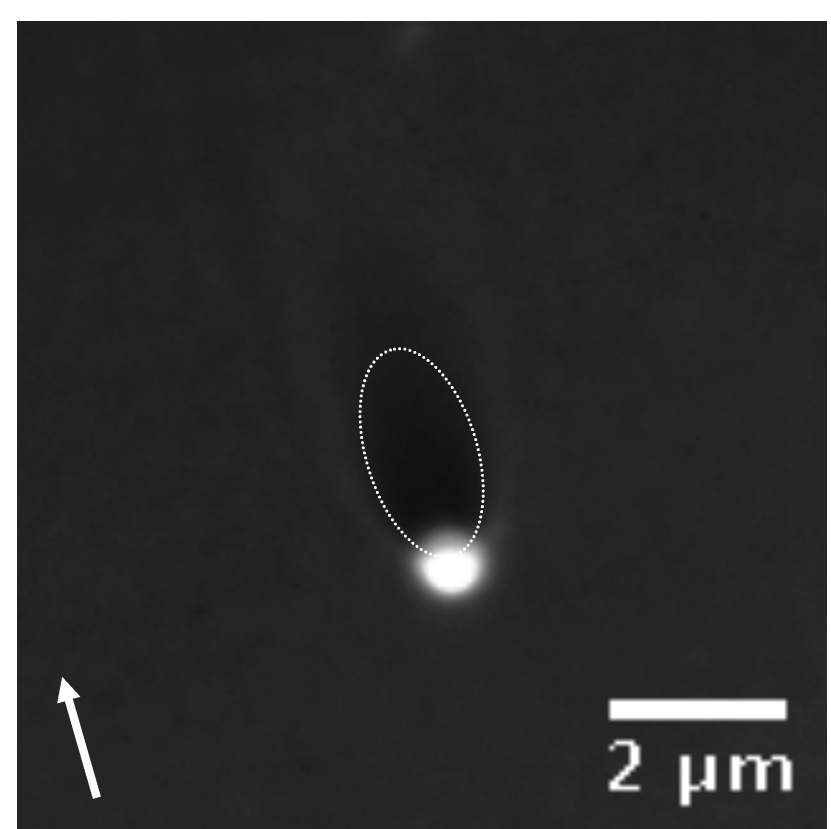

Figure 3. PEEM image of a dark brown human iridal stroma melanosome. The image clearly reveals the shadow generated as a result of absorption of the incident light. The shadow, marked by a white dashed line, is oriented in the direction of the incident light (indicated by the white arrow).

limit, we calculate the average path length assuming the melanosomes are oriented perpendicular to the direction of incident light. This is a reasonable approximation; as the aspect ratio decreases, the melanosomes approach a spherical shape.

\section{Results}

Figure 3 shows a PEEM image of a dark brown human iridal stroma melanosome. The white arrow indicates the direction of the incident light. The shadow observed in the image, marked by a dashed line, lies along this direction, as expected. Taking the experimental geometry into account (a $13^{\circ}$ angle between the surface plane and the incident light), an average melanosome height of $255 \mathrm{~nm}$ would produce a shadow that extends $\sim 1.1$ $\mu \mathrm{m}$ from the surface normal passing through the center of the melanosome. However, the observed shadow lengths for the iridal stroma melanosomes extend to $\sim 2.4 \mu \mathrm{m}$. This difference arises because of diffraction effects; the wavelength of the incident light is comparable to the size of the melanosome. Such diffraction effects have been noted in PEEM studies on other nanoscale systems. ${ }^{24}$

To disaggregate the effects of absorption and scattering within the shadow region, we determined the portion of the shadow region that is void of significant scattering contribution. Following the methodology outlined by Chelaru et al., ${ }^{24}$ diffraction will influence the intensity in the shadow region beginning at a distance from the melanosome corresponding to the first maximum in the diffraction pattern of regular Fraunhofer diffraction. Note, according to Babinet's principle, the Fraunhofer diffraction pattern for an opaque object is the same as it is for an aperture of the same shape except that the intensity distributions are inverted. ${ }^{25}$ Thus, calculating the dimensions of the first maximum diffraction disk from an elliptical aperture is equivalent to the dimension of the first minimum diffraction disk observed for opaque ellipsoidal melanosomes. 
TABLE 1: Relative Composition and Absorption Coefficient for Melanosomes Isolated from Different Colored Human Iridal Stroma Tissue ${ }^{a}$

\begin{tabular}{lccl}
\hline iride color & $\begin{array}{c}\text { eumelanin/ } \\
\text { pheomelanin }\end{array}$ & \% pheomelanin & $\varepsilon \mathrm{c} / \mathrm{cm}^{-1}$ \\
\hline dark brown & 14.8 & 6 & $4195(1400)$ \\
blue-green & 1.3 & 43 & $3160(900)$
\end{tabular}

${ }^{a}$ The standard deviation for the value of $\varepsilon c$ for the set of melanosomes examined is given in parentheses.

The intensity distribution for the illumination of an elliptical aperture with coherent plane waves is described by ${ }^{26}$

$$
I \propto\left[\frac{J_{1}(x)}{x}\right]^{2}
$$

where $J_{1}(x)$ is a first-order Bessel function and $x=2 \pi a \varepsilon p / \lambda z$, $2 a$ is the length of the major ellipsoidal axis, $\varepsilon$ is the ellipsoidal axial ratio $(b / a), p$ is the radius of the diffraction pattern produced on the plane of observation, $z$ is the distance between the aperture and the plane of observation, and $\lambda$ is the wavelength of the incident light. The first maximum of the diffraction pattern corresponds to the first zero of the function $J_{1}(x)$, occurring at $x=3.83166\left(m_{0}\right)$. Thus, the dimension of the first maximum observed on the plane of observation can be expressed as

$$
p=\frac{\lambda m_{0} z}{2 \pi a \varepsilon}
$$

This expression then gives the distance in the shadow region from the melanosome that is void of any significant contribution from scattering and thereby defines the region from which the absorption coefficient can be calculated. Under the experimental geometry, $z$ is equivalent to $2 b / \tan (\alpha)$ where $2 b$ is the length of the minor axis of the ellipse (the height of the melanosome from the surface plane) and $\alpha=13^{\circ}$, the angle between the surface plane and the incident light. With average ellipsoidal axes of $2 a=550 \mathrm{~nm}$ and $2 b=255 \mathrm{~nm}$ and $\lambda=244 \mathrm{~nm}$, the distance from the melanosomes that is void of significant scattering contributions is $\sim 2.6 \mu \mathrm{m}$. This region is exemplified by the white dashed line in Figure 3 and is contained within the first diffraction ring that is visible in the image. The average photoemission current is then determined for this region. Specifically, the upper threshold values of the image were set to visually segment the image into shadow and substrate features. Each region was reduced to an average value by averaging the intensities of the included pixels. These values, taken with the calculated average path length, are then used to determine the absorption coefficients presented in Table 1.

The absorption coefficients listed in Table 1 also indicate a range representing one standard deviation for the full data set collected on individual melanosomes. It is important to note, however, that this is not a measurement of error, but rather a result of the fact that all melanosomes are analyzed using the average ellipsoidal dimensions determined from SEM measurements. The limited resolution of the microscope ${ }^{27}$ precludes the possibility of determining the lengths of the two axes directly. The previously reported ${ }^{19}$ SEM analysis of the dimensions of these melanosomes reveal clear Gaussian-shaped distributions for the two axes; the full-width half-maximum of these distributions corresponds to $\sim 28 \%$ of the average length. It is a)
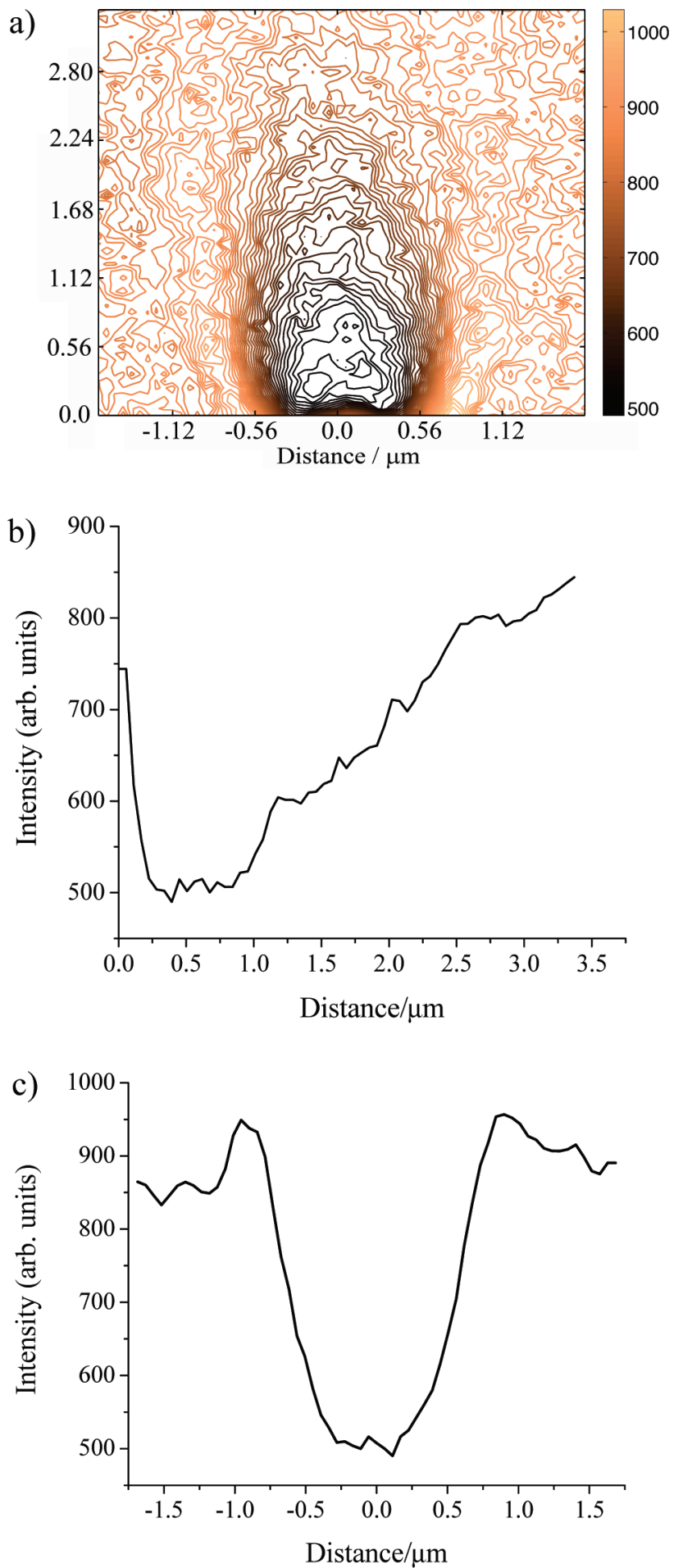

Figure 4. (a) Intensity contour plot of the shadow resulting from the dark brown human iridal stroma melanosome shown in Figure 3. Plots of the intensity of the image as a function of perpendicular distance from the center of the melanosome (b) and parallel to the melanosome at a distance of $0.56 \mu \mathrm{m}$ from its center (c).

the inability to characterize dimensions and orientation for each unique melanosome that contributes to the standard deviation reported.

An intensity contour plot of the shadow is shown in Figure 4a. Absorption of the melanosome is observed by the reduction of the photoemission intensity. Line plots reveal the spatial dependence of the photoemission intensity throughout the shadow. Plots of the intensity along the direction of incident light and perpendicular to the incident light are shown in Figure 
$4 \mathrm{~b}$ and $4 \mathrm{c}$, respectively. The ellipsoidal shape of the melanosome dictates the shape of these plots. The intensity is symmetric about the center of the melanosome. As one moves away from the center toward the ends of the long axis, the path length through the melanosome decreases causing more UV light to reach the surface and subsequently produce higher photoemission from the substrate behind the melanosome. Accordingly, as one moves away from the equator toward the top of the melanosome, the pathlengths through the melanosome decrease, and higher photoemission is observed in the corresponding shadow regions.

\section{Discussion}

At the outset, it is important to summarize three important common properties shared by the melanosomes from the different colored irides examined. First, they are ellipsoidal in overall shape, with a long axis of $\sim 550 \mathrm{~nm}$ and an aspect ratio of $\sim 2 .{ }^{19}$ Second, we previously presented atomic force microscopy data that demonstrated that these melanosomes consist of substructures that are $\sim 20-30 \mathrm{~nm}$ in diameter. ${ }^{19}$ This is common to this class of organelle and has been widely observed. ${ }^{28-30}$ Third, the outermost pigment surface of these melanosomes is pure eumelanin, despite the significant difference in their overall pigment composition, consistent with a "casing" model of mixed melanogenesis (the pheomelanin present in the melanosome is encased by eumelanin). , $, 6,31-34^{-}$ Therefore, with increased pheomelanin content, the outer eumelanin coating will be thinner.

In comparing the melanosomes from the dark brown and bluegreen irides, a $25 \%$ decrease in the absorption coefficient is observed for a 7-fold increase in the relative concentration of pheomelanin. It is difficult to determine a functional form for how the absorption coefficient depends on the relative concentrations of the two pigments from only two data points, but the data clearly demonstrate the absorption coefficient decreases with increasing pheomelanin content.

Consider the molecular origin of the decreased absorption of the melanosomes with increased pheomelanin content. In addition to pigment, melanosomes contain proteins, lipids, and various ions. However, in the case of human iridal stroma melanosomes, the quantities of each of these constituents are not known. Although several studies have examined the protein content of melanosomes isolated from different human and animal tissue sources, the majority of these were based on melanosomes that had been isolated with acid/base extraction techniques. ${ }^{35-37}$ This particular extraction method was widely used to extract melanosomes from tissue, but this approach is now known to both alter the physical and biological properties of the melanosome and result in isolation of a larger protein content than that associated with intact melanosomes. ${ }^{29}$

As a specific example, melanin comprises $\sim 86 \%$ of the material present in enzymatically extracted melanosomes from black human hair, whereas the acid-base extraction of melanosomes from the same hair sample alters the melanosome structure, and the isolated materials contain $40-52 \%$ protein, depending on the method used. ${ }^{29}$ Thus, although previous isolation techniques produced samples with a large protein content, enzymatic isolation of intact melanosomes reveals that the dominant constituent in the organelle is the melanin pigment.

In addition, most lipids and amino acids exhibit negligible absorption at $\lambda=244 \mathrm{~nm}$. Aromatic amino acids, however, are an exception to this general statement, and have absorption cross sections from $\sim 40$ (phenylalanine) to $3000 \mathrm{M}^{-1} \mathrm{~cm}^{-1}$ (tryptophan), the latter being similar to the cross sections of melanin monomers. ${ }^{38}$ These aromatic amino acids make up less than $5 \%$ of the proteins in bovine iride melanosomes, ${ }^{39}$ and given that the relative melanin to protein content is on the order of $7: 1$, the presence of these aromatic amino acids is expected to contribute at most $0.7 \%$ of the total absorption at $\lambda=244 \mathrm{~nm}$. This is a negligible contribution. Thus, the absorption does not reflect lipid and protein content. We therefore conclude that the decrease in absorption observed with increasing pheomelanin content must arise from the changing composition of the constituent pigments.

In the case of eumelanin, the molecular chromophores are generated from the monomeric dihydroxyindole building blocks DHI and DHICA. The literature values for the absorption cross sections of DHI and DHICA vary, depending on the solvent, $\mathrm{pH}$, or both; representative values are 1720 (in deaerated water) and $6590 \mathrm{M}^{-1} \mathrm{~cm}^{-1}$ (in $\mathrm{NaOH}$ solution), respectively. ${ }^{40-42}$ Both molecules exhibit larger absorption cross sections in phosphate buffer (4485 and $8205 \mathrm{M}^{-1} \mathrm{~cm}^{-1}$ for DHI and DHICA, respectively). Thus, if the DHI/DHICA ratio were to change for the eumelanin present in the melanosomes from different colored irides, then the data in Table 1 could reflect changes in the eumelanin pigment. ${ }^{43,44}$ Wielgus and Sarna address the DHI/ DHICA ratio of colored human irides through their reported chemical degradation analyses. ${ }^{45}$ Hydrogen peroxide degradation of melanosomes from several different colored irides yields a fairly constant PTCA/PDCA ratio, reflective of a constant DHI/ DHICA ratio for melanosomes isolated from different colored irides. Thus, the change in the observed absorption coefficients is representative of the relative contributions of the two different pigments, pheomelanin and eumelanin.

The chromophore in pheomelanin is built from molecules such as dihydro-1,4-benzothiazine-3-carboxylic acid (DHBTCA) and 6-(2amino-2-carboxyethyl)-4-hydroxy-benzothiazole (BZ). ${ }^{46}$ Ito and coworkers characterized the absorption properties of the pheomelanin precursors DHBTCA and BZ and found that these molecules have absorption cross sections of 8570 and $7250 \mathrm{M}^{-1} \mathrm{~cm}^{-1}$ in a $0.1 \mathrm{M}$ $\mathrm{HCl}$ solution. Taken with the absorption cross sections reported above for DHI and DHICA, we conclude that the monomeric precursors to eumelanin and pheomelanin have similar UV absorption cross sections. However, these precursors have different molecular volumes. AM1 calculations of the molecular volumes of DHI, DHICA, BZ, and DHBTCA give 0.129, 0.172, 0.195, and $0.268 \mathrm{~nm}^{3}$, respectively (http://www.molinspiration.com/services/ volume.html). The chemical degradation analyses used to determine the eumelanin and pheomelanin content is a measure of the number of these monomer building blocks present in the intact pigment, and for a constant total volume, an increase in the number of DHBTCA units must result in a loss of a larger number of DHI/ DHICA units because DHBTCA occupies a larger volume than a DHI/DHICA molecule. Therefore, with increasing pheomelanin content, the total number of monomeric chromophores (eumelanin and pheomelanin combined) decreases.

Taking the volume of the melanosome (calculated from the average dimensions determined from the SEM size analysis), the eumelanin/pheomelanin ratio determined from chemical analyses, and the above volumes for the molecular building blocks (using an average of the DHI/DHICA volumes to represent the eumelanin precursors), we find that there is a $32 \%$ decrease in the number of chromophores in blue-green iridal stroma melanosomes as compared with dark brown iridal melanosomes. This is in good agreement with the measured absorption decrease (25\%). We would conclude from this calculation that the dominant contribution to the decreased absorption of the melanosome with increasing pheomelanin 


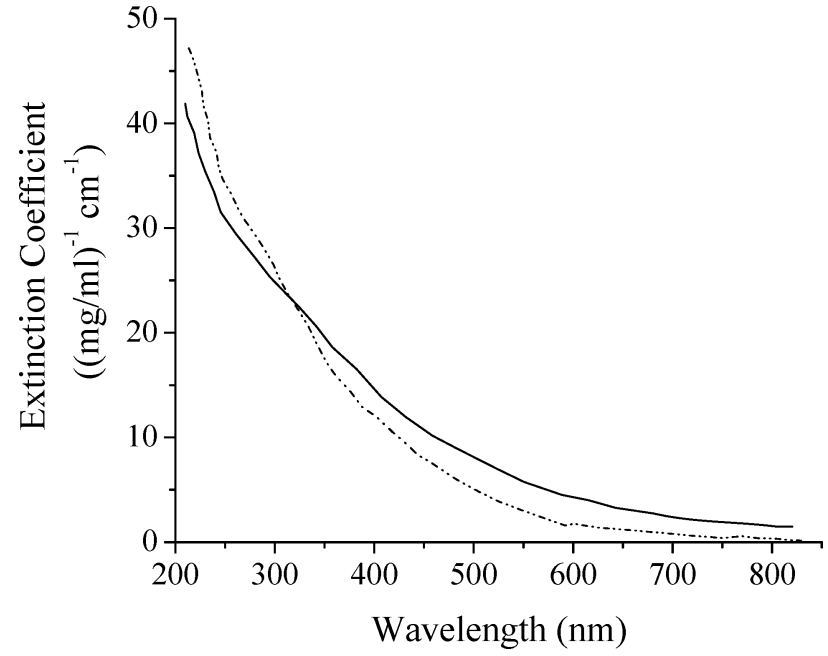

Figure 5. Absorption spectra for synthetic eumelanin (solid line) and pheomelanin (dashed line). Data were obtained from ref 9. The spectra reveal an absorption ratio of 1.13 for pheomelanin/eumelanin at 244 $\mathrm{nm}$, which is excellent agreement with the value of 1.2 obtained from the analysis of the absorption coefficients of the intact melanosomes.

content results from a decrease in the number of absorbing chromophores. Taking the decrease in number of chromophores into account, and the absorption data for the intact melanosomes given in Table 1, we would predict that the absorption of pheomelanin at $\lambda=244 \mathrm{~nm}$ is a faculty of 1.2 greater than that of eumelanin, which is also consistent with the absorption cross sections of the constituent monomers.

Currently, it is only possible to collect quantitative absorption spectra of melanins for soluble synthetic samples. Although synthetic samples are commonly studied, the spectra of the pigments depend on several factors, including the synthetic method used and the oxidation/reduction state of various sites within the resulting polymer. ${ }^{47}$ Sarna and co-workers ${ }^{9}$ reported absorption spectra for synthetic eumelanin and pheomelanin, and when these spectra are plotted as a function $\mathrm{cm}^{-1}(\mathrm{mg} /$ $\mathrm{mL})^{-1}$ vs $\lambda$ (Figure 5), they find that the pheomelanin/eumelanin absorption at $\lambda=244 \mathrm{~nm}$ is $\sim 1.13$, which is in excellent agreement with our determination of 1.2 from the analysis of the absorption coefficients of the intact melanosomes. This further confirms that the optical properties of the synthetic and natural pigments are similar and that in the melanosome, the absorption coefficient for pheomelanin is less than for eumelanin at wavelengths of biological relevance $(\sim 315-400 \mathrm{~nm})$.

Consider the implications of this result. The relative amounts of pheomelanin and eumelanin in tissue varies, and the factors controlling their presence have become increasingly important to understand because the epidemiology of skin and ocular cancers suggests that increasing relative proportions of pheomelanin correlates with an increased risk factor for these diseases. ${ }^{10,11,48}$ These epidemiological observations have spurred a significant effort aimed at determining whether pheomelanin plays a causative role in these cancers. To date, no definitive view has been provided because there is currently data that both supports $^{12,13,17,23,48-50}$ and refutes ${ }^{51-55}$ pheomelanin's role. However, the work described herein offers a context to reexamine this debate from results based on intact melanosomes. The present work indicates that the photoprotective role of the melanosomes is compromised with increased pheomelanin content as the absorption coefficient at biologically relevant wavelengths is reduced. The correlation between epidemiological data and the eumelanin/pheomelanin ratio may then reflect a decreased ability to attenuate biologically relevant wavelengths, resulting in exposure of the underlying tissues to UV light accompanying the increasing pheomelanin content in the melanosome. Further progress in this area depends on determining the quantum efficiency for oxygen activation and further exploring the wavelength dependence of the absorption coefficients of both pigments from studies on intact natural melanosomes.

Acknowledgment. This work is supported by Duke University. We thank Professor Yongan Tang for the simulation of the interaction of the electromagnetic field with an opaque ellipsoidal object. We would also like to thank Dr. Robert Guenther for the many helpful discussions about the diffraction patterns produced from elliptical apertures.

\section{References and Notes}

(1) Ito, S.; Wakamatsu, K. Chemistry of Melanins. In The Pigmentary System: Physiology and Pathophysiology; 2nd ed.; Norlund, J. J., Boissy, R. E., Hearing, V. J., King, R. A., Oetting, W. S., Ortonne, J.-P., Eds.; Blackwell: Malden, 2006; pp 282-310.

(2) Simon, J. D.; Peles, D.; Wakamatsu, K.; Ito, S. Pigm. Cell Melanoma Res. 2009, 22, 563-579.

(3) Hearing, V. J. Pigm. Cell Res. 2000, 13, 23-34.

(4) Meredith, P.; Sarna, T. Pigm. Cell Res. 2006, 19, 572-594.

(5) Ito, S. Pigm. Cell Res. 2003, 16, 230-236.

(6) Ito, S.; Wakamatsu, K. Photochem. Photobiol. 2008, 84, 582-592.

(7) Prota, G. Pigm. Cell Res. 2000, 13, 283-293.

(8) Liu, Y.; Hong, L.; Wakamatsu, K.; Ito, S.; Adhyaru, B.; Cheng, C.-Y.; Bowers, C. R.; Simon, J. D. Photochem. Photobiol. 2005, 81, 135144.

(9) Sarna, T.; Swartz, H. M. The Physical Properties of Melanins. In The Pigmentary System; Nordlund, J. J., Boissy, R. E., Hearing, V. J., King, R. A., Ortonne, J. P., Eds.; Oxford University Press: New York, 1998; pp $333-357$.

(10) Wakamatsu, K.; Hu, D.-N.; McCormick, S. A.; Ito, S. Pigm. Cell Res. 2007, 21, 97-105.

(11) Hu, D. N.; Simon, J. D.; Sarna, T. Photochem. Photobiol. 2008, $84,639-644$.

(12) Ye, T.; Hong, L.; Garguilo, J.; Pawlak, A.; Edwards, G. S.; Nemanich, R. J.; Sarna, T.; Simon, J. D. Photochem. Photobiol. 2006, 82, 733-737.

(13) Takeuchi, S.; Zhang, W.; Wakamatsu, K.; Ito, S.; Hearing, V. J.; Kraemer, K. H.; Brash, D. E. Proc. Natl. Acad. Sci. U.S.A. 2004, 101, 15076-15081.

(14) Persad, S.; Menon, I. A.; Haberman, H. F. Photochem. Photobiol. 1983, 37, 63-68.

(15) Harsanyi, Z. P.; Post, P. W.; Brinkmann, J. P.; Chedekel, M. R.; Deibel, R. M. Experientia 1980, 36, 291-292.

(16) Chedekel, M. R.; Smith, S. K.; Post, P. W.; Pokora, A.; Vessell, D. L. Proc. Natl. Acad. Sci. U.S.A. 1978, 75, 5395-5399.

(17) Ye, T.; Pawlak, A.; Sarna, T.; Simon, J. D. Photochem. Photobiol. 2008, 84, 437-443.

(18) Peles, D.; Simon, J. D. Photochem. Photobiol. 2010, 86, 279-281.

(19) Peles, D. N.; Hong, L.; Hu, D. N.; Ito, S.; Nemanich, R. J.; Simon,

J. D. J. Phys. Chem. B 2009, 113, 11346-11351.

(20) Prota, G.; Hu, D. N.; Vincensi, M. R.; McCormick, S. A.; Napolitano, A. Exp. Eye Res. 1998, 67, 293.

(21) Liu, Y.; Hong, L.; Wakamatsu, K.; Ito, S.; Adhyaru, B. B.; Cheng, C.-Y.; Bowers, C. R.; Simon, J. D. Photochem. Photobiol. 2005, 81, 510.

(22) Ade, H.; Yang, W.-C.; English, S. L.; Hartman, J.; Davis, R. F.; Nemanich, R. J.; Litvinenko, V. N.; Pinayev, I. V.; Wu, Y.; Madey, J. M. J. Surf. Rev. Lett. 1998, 5, 1257.

(23) Samokhvalov, A.; Hong, L.; Liu, Y.; Garguilo, J.; Nemanich, R. J.; Edwards, G. S.; Simon, J. D. Photochem. Photobiol. 2005, 81, 145-148. (24) Chelaru, L. I.; Hoegen, M. H.-V.; Thien, D.; Heringdorf, F.-J. M. Phys. Rev. B 2006, 73, 115416.

(25) Guenther, R. D. Fresnel Diffraction. In Modern Optics; John Wiley \& Sons: New York, 1990; p 463.

(26) Kathuria, Y. P. IEEE Trans. Antennas Propag. 1983, AP-31, 360.

(27) Peles, D. N.; Simon, J. D. Photochem. Photobiol. 2009, 85, 8.

(28) Clancy, C. M. R.; Simon, J. D. Biochemistry 2001, 40, 1335313360 .

(29) Liu, Y.; Kempf, V. R.; Nofsinger, J. B.; Weinert, E. E.; Rudnicki, M.; Wakamatsu, K.; Ito, S.; Simon, J. D. Pigm. Cell Res. 2003, 16, 355365 .

(30) Liu, Y.; Simon, J. D. Pigm. Cell Res. 2003, 16, 606-618. 
(31) Ozeki, H.; Ito, S.; Wakamatsu, K.; Ishiguro, I. Biochim. Biophys. Acta 1997, 1336, 539-548.

(32) Agrup, G.; Hansson, C.; Rorsman, H.; Rosengren, E. Arch. Dermatol. Res. 1982, 272, 103-115.

(33) Bush, W. D.; Garguilo, J.; Zucca, F. A.; Albertini, A.; Zecca, L.; Edwards, G. S.; Nemanich, R. J.; Simon, J. D. Proc. Natl. Acad. Sci. U.S.A. 2006, 103, 14785-14789.

(34) Ito, S. Proc. Natl. Acad. Sci. U.S.A. 2006, 103, 14647-14648.

(35) Sarna, T.; Froncisz, W.; Hyde, J. S. Arch. Biochem. Biophys. 1980, $202,304$.

(36) Borovanský, J.; Duchoň, J. Dermatologica 1974, 149, 116.

(37) Borovanský, J.; Hach, P. Neoplasma 1973, 20, 325.

(38) Cantor, C. R.; Schimmel, P. R. Biophysical Chemistry; W. H. Freeman: New York, 1980; Vol. 2, p 377.

(39) Ward, W.; Simon, J. D. Pigm. Cell Res. 2007, 20, 61-69.

(40) Murphy, B. P.; Schlutz, T. M. J. Org. Chem. 1985, 50, 2790-2791.

(41) Aroca, P.; Solano, F.; Garcie-Borron, J. C.; Lozano, J. A. J. Biochem. Biophys. Methods 1990, 21, 35-46.

(42) Benigni, J. D.; Minnis, R. J. Heterocycl. Chem. 1965, 2, 387-392.

(43) Wakamatsu, K.; Ito, S. Pigm. Cell Res. 2002, 15, 174-183.

(44) Ozeki, H.; Ito, S.; Wakamatsu, K. Pigm. Cell Res. 1996, 9, 51-57.

(45) Wielgus, A. R.; Sarna, T. Pigm. Cell Res. 2005, 18, 454-464.

(46) Wakamatsu, K.; Ohtara, K.; Ito, S. Pigm. Cell Melanoma Res. 2009, $22,474-486$
(47) Sarna, T. J. Photochem. Photobiol., B 1992, 12, 215.

(48) Vincensi, M. R.; d'Ischia, M.; Napolitano, A.; Procaccini, E. M.; Riccio, G.; Monfrecola, G.; Santoianni, P.; Prota, G. Melanoma Res. 1998, $8,53-58$.

(49) Wenczl, E.; Van der Schans, G. P.; Roza, L.; Kolb, R. M.; Timmerman, A. J.; Smit, N. P. M.; Pavel, S.; Schothorst, A. A. J. Invest. Dermatol. 1998, 111, 678-682.

(50) Wenczl, E.; van der Schans, G. P.; Roza, L.; Kolb, R.; Smit, N.; Schothorst, A. A. J. Invest. Dermatol. 1998, 110, 693-693.

(51) Sarna, T.; Sealy, R. C. Photochem. Photobiol. 1984, 39, 69-74.

(52) Hennessy, A.; Oh, C.; Diffey, B.; Wakamatsu, K.; Ito, S.; Ree., J. Pigm. Cell Res. 2005, 18, 220-223.

(53) Sarna, T.; Menon, I. A.; Sealy, R. C. Photochem. Photobiol. 1985, $42,529-532$.

(54) Sealy, R. C.; Sarna, T.; Wanner, E. J.; Reszka, K. Photochem. Photobiol. 1984, 40, 453-459.

(55) Sarna, T.; Menon, I. A.; Sealy, R. C. Photochem. Photobiol. 1984, $39,805-809$.

(56) Taflove, A.; Hagness, S. C. Computational Electrodynamics: The Finite-Difference Time-Domain Method; Artech: Norwood, 2000.

(57) Oskooi, A. F.; Roundy, D.; Ibanescu, M.; Bermel, P.; Joannopoulos, J. D.; Johnson, S. G. Comput. Phys. Commun. 2010, 181, 687.

JP102603B 